\title{
Propagação de compostos da gasolina em solos: 1. Zona não saturada
}

\section{Propagation of the gasoline compounds in the soil: 1. Unsaturated zone}

\section{Edson Abel dos Santos Chiaramonte}

Engenheiro Químico pela Universidade Federal do Rio Grande do Sul (UFRGS) - Porto Alegre (RS), Brasil. Mestre em Engenharia Química pela Universidade Federal do Rio de Janeiro (UFRJ) - Rio de Janeiro (RJ), Brasil. Doutor em Engenharia Mecânica pela UFRGS. Professor Adjunto do curso de Engenharia Química da Universidade Federal do Pampa (UNIPAMPA) - Bagé (RS), Brasil.

\section{Rubens Müller Kautzmann}

Engenheiro de Minas pela UFRGS. Mestre e Doutor em Engenharia pela UFRGS - Porto Alegre (RS), Brasil. Professor Adjunto do curso de Engenharia Ambiental da Unilassale - Canoas (RS), Brasil.

\section{Carlos Hoffman Sampaio}

Engenheiro de Minas pela UFRGS. Mestre em Engenharia de Minas pela UFRGS - Porto Alegre (RS), Brasil. Doutor em Processamento Mineral pela Rheinisch Westfaelische Technische Hochschule Aachen - Aachen, Germany. Professor Adjunto da UFRGS - Porto Alegre (RS), Brasil.

\section{Jorge Rodolfo Silva Zabadal}

Engenheiro Químico pela UFRGS. Mestre e Doutor em Engenharia Mecânica pela UFRGS - Porto Alegre (RS), Brasil. Professor Adjunto do Departamento de Engenharia Mecânica da UFRGS - Porto Alegre (RS), Brasil.

\section{João Alifantes}

Bacharel em Química pela UFRGS. Mestre e Doutor em Química pela UFRGS - Porto Alegre (RS), Brasil. Professor Adjunto do Curso de Engenharia de Bioprocessos e Biotecnologia da UERGS - Novo Hamburgo (RS), Brasil.

\section{Renato Letizia Garcia}

Engenheiro Químico pela UFRGS. Mestre em Recursos Hídricos e Saneamento Ambiental pela UFRGS - Porto Alegre (RS), Brasil. Doutor em Engenharia Mecânica pela UFRGS. Professor Assistente da disciplina de Engenharia de Bioprocessos e Biotecnologia da UERGS - Novo Hamburgo (RS), Brasil.

\section{Resumo}

Esse trabalho descreve o estudo da propagação de tolueno na zona não saturada do solo. Mostram-se o procedimento experimental desenvolvido em colunas de laboratório, os resultados experimentais obtidos, uma análise dos resultados aplicando-se modelagem matemática, a análise estatística aplicada ao denominado modelo de puffs de simulação da dispersão e uma discussão do prosseguimento desse trabalho.

Palavras-chave: propagação; gasolina; solo; experimentos; modelagem.

\section{Abstract}

This work describes the study of the propagation of toluene in the unsaturated zone of the ground. It is shown the experimental procedure in laboratory columns. The gotten experimental results and their analysis with a mathematical modeling are shown. Also is shown an analysis statistics applied to the called the puffs' models of the dispersion simulation and a quarrel of the continuation of this work.

Keywords: propagation; gasoline; soil; experiments; modeling. 


\section{Introdução}

Os derramamentos de produtos químicos no solo causam danos ao ambiente do solo e são nocivos aos seres vivos e a sua flora. Entre esses vazamentos, os de frações do petróleo (gasolina, diesel, querosene, nafta) que ocorrem durante a produção, a transformação, o transporte e a distribuição têm chamado a atenção nos últimos anos, conforme os trabalhos de Santos (1998), Cordazzo (2000) e Penner (2000). Portanto, é importante o conhecimento da distribuição desses produtos no solo e os mecanismos envolvidos em sua propagação: a difusão, a advecção e a degradação natural, conforme Nunes e Corseuil (2007). Também, são importantes as aplicações dos modelos matemáticos que realizam a estimativa dessa propagação nos procedimentos de avaliação de impacto ambiental (FÁVERO, MUNIZ; SANTOS, 2007). Neste contexto, o grupo de pesquisa investiga através de estudos experimentais e modelagem matemática esta propagação nas zonas não saturada e saturada do solo. O transporte e a difusão de compostos hidrocarbonetos no solo envolve os fenômenos físicos como escoamento por gravidade, lixiviação, difusão mecânica e molecular. Os fenômenos de distribuição dos compostos entre as fases: sólida, ar, água e óleo (adsorção na fase sólida, solubilidade na fase líquida, e transferência de massa para a fase vapor) e processos de degradação (química e biológica) também estão presentes. Nesta etapa da pesquisa, investigam-se os fenômenos de dispersão na zona não saturada, segundo Jury, Gardner e Gardner (2005) e Domênico e Schwartz (1997). Dados experimentais estão sendo obtidos numa coluna de solo em escala de laboratório. O composto tolueno é usado como representativo da gasolina e valores quantitativos de concentração são obtidos ao longo da coluna. Este artigo contém os seguintes itens: a dispersão de compostos na zona não saturada do solo, os materiais e os métodos para a obtenção de dados experimentais, os resultados experimentais, uma análise dos resultados usando-se modelagem matemática e a conclusão desta etapa do trabalho.

\section{Propagação de compostos na zona não saturada do solo}

O transporte e a difusão de compostos hidrocarbonetos no solo envolve os fenômenos físicos de propagação como escoamento por gravidade, lixiviação, difusão mecânica e molecular, conforme Abriola e Pinder (1985). Os compostos também sofrem fenômenos de interação entre as fases e processos de degradação no solo. A região do solo em que o componente está se propagando também é um fator importante na modelagem da dispersão. O modelo deve levar em conta se é a fase não saturada ou saturada do solo. As primeiras camadas do solo são preenchidas por ar, umidade, e a fase orgânica nos seus vazios (zona não saturada).

Na zona nãosaturada, o espaço poroso é preenchido pela somatória dos fluidos presentes, como apresentado na Equação 1.

$$
\theta=\theta_{w}+\theta_{a r}+\theta_{o}
$$

Onde:

$\theta$ : porosidade do solo;

$\theta_{\mathrm{w}}$ : fração de vazios ocupados pela água;

$\theta_{\mathrm{ar}}$ : fração de vazios ocupados pelo ar;

$\theta_{\mathrm{o}}$ : fração de vazios ocupados pela fase líquida não aquosa (NAPL) ou óleo (o)

Então, a concentração total do componente "i" no solo é dada pela relação apresentada na Equação 2:

$C_{i, t}=\theta_{w} C_{i, w}+\theta_{a r} C_{i, a r}+\theta_{o} C_{i, o}+\rho_{b} C_{i, s}$

Onde:

$\mathrm{C}_{\mathrm{i}, \mathrm{t}}$ : concentração total de "i" no solo, em ( $\left.\mathrm{g}_{\text {de uip }}\right)\left(\mathrm{m}^{-3}{ }_{\text {de solo }}\right)$;

$\rho_{\mathrm{b}}$ : densidade do solo, em ( $\left.\mathrm{g}_{\text {de sólido }}\right)\left(\mathrm{m}^{-3}\right.$ de solo $)$;

$\mathrm{C}_{\mathrm{i}, \mathrm{w}}$ : concentração do componente " $\mathrm{i}$ " na fase água, em $\left(\mathrm{g}_{\text {de } \mathrm{u}_{\mathrm{i}} \text { " }}\right)\left(\mathrm{m}^{-3}\right.$ de água $)$; $\mathrm{C}_{\mathrm{i}, \mathrm{ar}}$ : concentração do componente "i" na fase vapor, em $\left(\mathrm{g}_{\text {de }} \mathrm{u}_{\mathrm{i} \text { " }}\right)\left(\mathrm{m}^{-3}\right.$ de ar $)$; $\mathrm{C}_{\mathrm{i}, \mathrm{o}}$ : concentração do componente "i" na fase óleo, em ( $\left.\mathrm{g}_{\text {de " wi }}\right)\left(\mathrm{m}_{\text {de óleo }}^{-3}\right)$; $\mathrm{C}_{\mathrm{i}, \mathrm{s}}$ : concentração do componente "i" na fase sólida, em $\left(\mathrm{g}_{\text {de wip }}\right)\left(\mathrm{g}^{-1}\right.$ de sólido $)$.

Considerando a não formação da fase de óleo na camada de solo e o mecanismo de propagação predominante, do composto orgânico, ser a difusão molecular no ar,a equação da difusão (Equação 3), que descreve a propagação unidimensional na zona nãosaturada do solo, conforme Grathwohl et al. (2003) e Fetter (1998), é escrita na forma:

$\frac{\partial C_{i, a r}}{\partial t}=\frac{D_{E}}{\alpha} \frac{\partial^{2} C_{i, a r}}{\partial z^{2}}$

Onde:

t: tempo de liberação do composto;

z: distância na direção vertical;

$\mathrm{D}_{\mathrm{E}}$ : coeficiente de difusão efetivo na fase ar do solo.

O coeficiente de difusão é estimado pela relação de Millington e Quirk (1961) - Equações 4 e 5.

$D_{E}=D_{i, a r} \frac{\left(\theta_{a r}\right)^{\frac{10}{3}}}{\theta^{2}}$

Onde:

$\mathrm{D}_{\mathrm{i}, \mathrm{ar}}$ : coeficiente de difusão do composto no ar.

$\alpha=\theta_{a r}+\frac{\theta_{w}}{K_{h}}+\frac{K_{d} \rho_{b}}{K_{h}}$

Onde:

a: parâmetro que leva em conta a distribuição do composto nas fases ar, água e material sólido do solo;

$\mathrm{K}_{\mathrm{h}}$ : constante da lei de Henry (adimensional);

$\mathrm{K}_{\mathrm{d}}$ : coeficiente de distribuição entre a fase sólida e a fase água. 
Nos estudos de Engenharia em análise de risco são usados modelos matemáticos de propagação em meio semi-infinito. Conforme Behrendt (1996), a seguinte solução analítica é usada para uma liberação instantânea de um composto no solo (Equação 6).

$C_{i, a r}(z, t)=\frac{C_{i, o}}{2}\left\{\operatorname{erf}\left(\frac{h-z}{2 \sqrt{D_{E} t}}\right)+\operatorname{erf}\left(\frac{h+z}{2 \sqrt{D_{E} t}}\right)\right\}$

Onde:

$\mathrm{C}_{\mathrm{i}, \mathrm{o}}$ : valor inicial da concentração do composto;

H: valor inicial da concentração da espessura da liberação;

Erf (): função erro.

Se a liberação apresentar característica de uma liberação contínua (um vazamento que possua um tempo de elevado) pode ser usada a seguinte solução analítica (Equação 7):

$C_{i, a r}(z, t)=C_{i, o} \operatorname{erfc}\left(\frac{z}{2 \sqrt{D_{E} t}}\right)$

Nesse trabalho, o meio de propagação é limitado ao comprimento das colunas de laboratório, mostrado na Figura 1, e a solução matemática é aplicada a esse contorno limitado, conforme Ozisik (1993). São usadas duas soluções analíticas: a primeira com a condição de contorno de concentração fixa no topo da coluna (ponto de liberação; $z=0$ ) e a segunda usa a condição de inexistência de fluxo no topo e as duas aplicam a condição de fluxo de massa no fundo da coluna. As seguintes soluções matemáticas em série são obtidas:

Solução para a condição de concentração fixa (Equação 8);

$C_{i, a r s}(z, t)=2 C_{i, 0} \sum_{m=1}^{\infty} e^{-D_{E}^{2} B_{m} t} \frac{B_{m}^{2}+H_{2}^{2}}{L\left(B_{m}^{2}+H_{2}^{2}\right)+H_{2}} \frac{\left(\cos \left(B_{m} L\right)-1\right)}{B_{m}} \operatorname{sen}\left(B_{m} z\right)$

Onde:

$\mathrm{B}_{\mathrm{m}}$ : raízes da Equação 9.

$B_{m} \cot \left(B_{m} L\right)-H_{2}=0$

Onde:

$\mathrm{H}_{2}$ : razão entre o coeficiente de transferência de massa no fundo da coluna e o coeficiente de difusão;

L: comprimento da coluna.

A solução (liberação intermitente) para não existência de fluxo é (Equação 10):

$C_{i, a r s}(z, t)=2 C_{i, 0} \sum_{m=1}^{\infty} e^{-D_{E}^{2} A_{m} t} \frac{A_{m}^{2}+H_{2}^{2}}{\left[L\left(A_{m}^{2}+H_{2}^{2}\right)+H_{2}\right] A_{m}} \operatorname{sen}\left(A_{m} L_{i}\right) \cos \left(A_{m} z\right)$

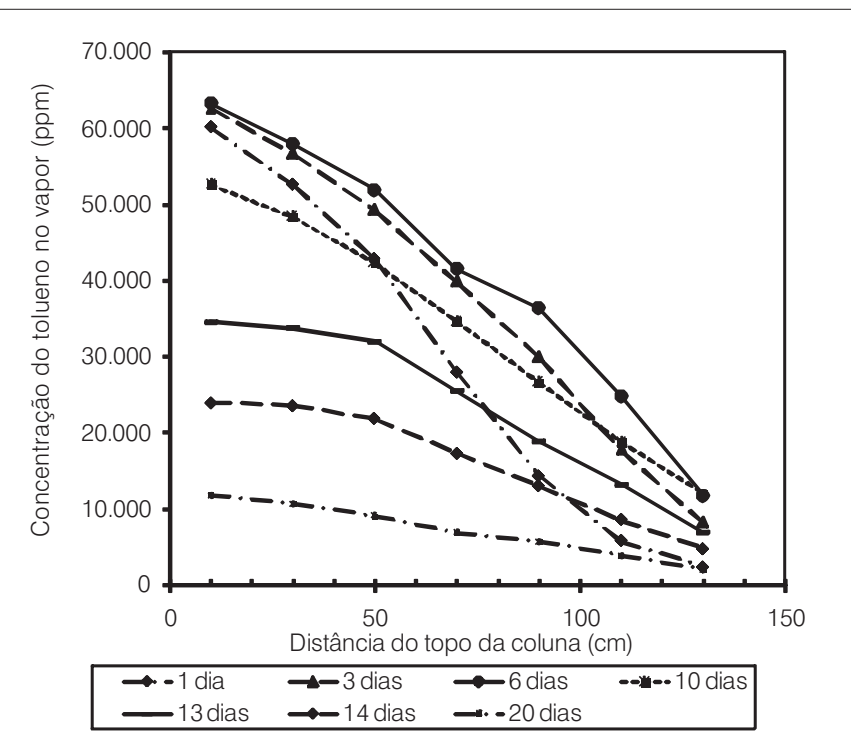

Figura 1 - Perfis de concentração de tolueno em coluna de 1,5 m.

Onde:

$\mathrm{A}_{\mathrm{m}}$ : raízes da Equação 11

$A_{m} \tan \left(A_{m} L\right)-H_{2}=0$

Onde:

$\mathrm{L}_{\mathrm{i}}$ : comprimento da liberação no ponto de emissão.

\section{Metodologia}

\section{Elaboração das soluções padrões}

Uma quantidade de tolueno é diluída em frasco contendo ar (frasco de volume conhecido) para a elaboração de misturas gasosas de concentrações conhecidas em parte por milhão (ppm) de massa; a curva padrão é obtida com o uso da técnica de cromatografia gasosa.

\section{Procedimento de liberação experimental na coluna de solo}

Uma quantidade do composto tolueno é liberada na coluna de solo. Depois de determinado período de tempo, realiza-se a coleta em várias alturas da fração fluida do solo. Em seguida, é determinada a concentração do composto no solo usando-se o método analítico de cromatografia gasosa.

\section{Materiais e equipamentos}

A coluna é um tubo de aço, com diâmetro de $5 \mathrm{~cm}$ e altura de $150 \mathrm{~cm}$, preenchida com areia fina visando simular um solo em 


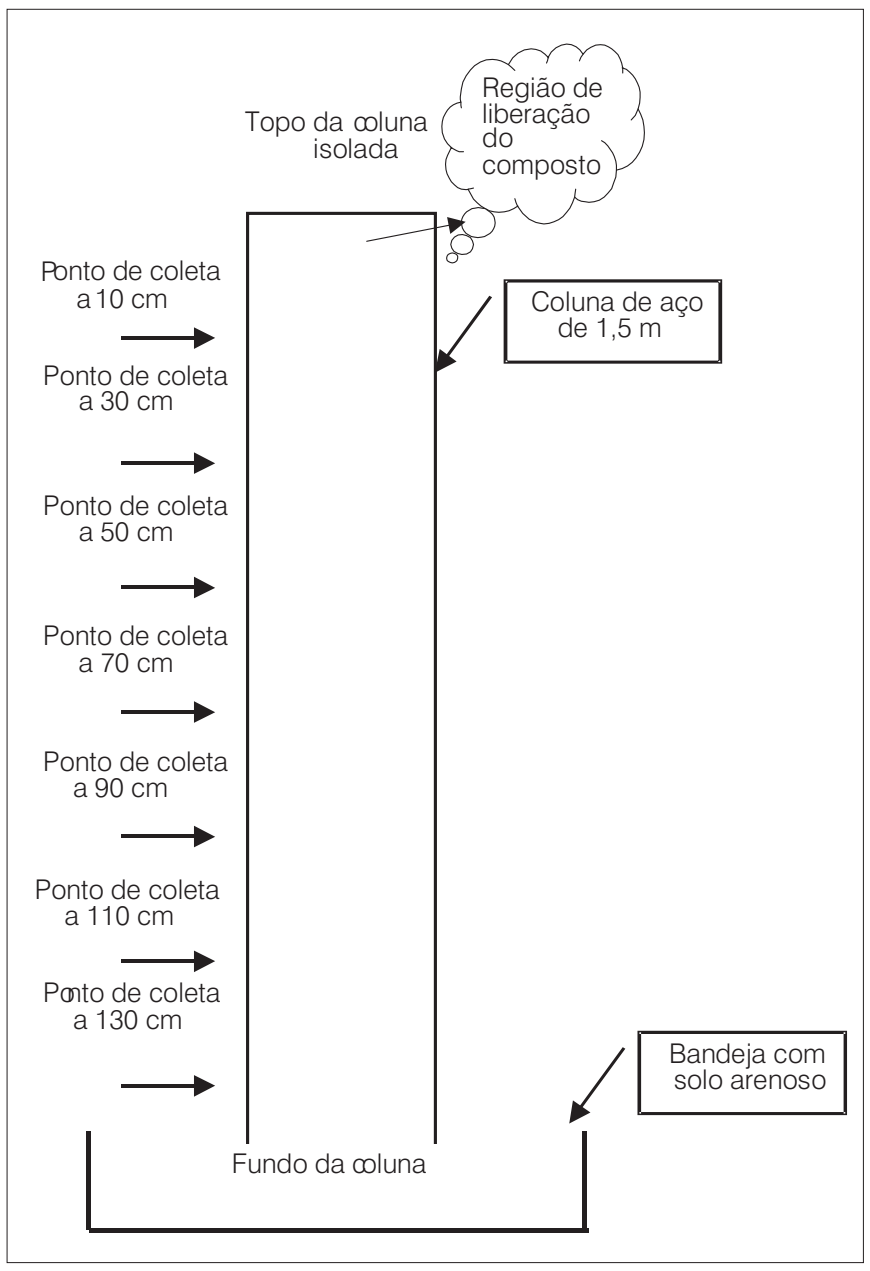

Figura 2 - Esquema da coluna de laboratório.

laboratório, conforme o esquema da Figura 2. A coluna apresenta 7 pontos de coleta de amostra espaçados $20 \mathrm{~cm}$ entre si. O reagente tolueno P.A. foi usado como composto representativo de um vazamento. Microsseringa de $10 \mu \mathrm{L}$ e pipeta de $1 \mathrm{~mL}$ também foram utilizadas. Cromatógrafo gasoso com detector por ionização de chama e coluna polar, estufa e balança analítica foram os equipamentos usados.

\section{Procedimento experimental}

O procedimento experimental consistiu das seguintes etapas:

1. Preenchimento da coluna de bancada (diâmetro de $5 \mathrm{~cm}$ e comprimento de 1,5 m) com areia fina;

2. Liberação de uma quantidade definida de tolueno, representando um composto volátil da gasolina (desejava-se obter uma liberação instantânea);

3. Coleta diária das amostras da fase vapor do solo nos sete pontos da coluna. A amostra era composta da mistura de tolueno e ar atmosférico sem a presença da fase sólida;
4. Determinação da concentração quantitativa do contaminante na amostra. Utilizou-se o procedimento analítico de cromatografia gasosa.

\section{Resultados}

Os valores experimentais, de concentração do tolueno na fase vapor, foram amostrados com uma coleta por dia. Esses valores foram obtidos, na coluna de $150 \mathrm{~cm}$ de comprimento, nos pontos de distância de 10, 30, 50, 70, 90, 110 e $130 \mathrm{~cm}$ medidos a partir do topo da coluna. A coluna é soldada no topo, ou seja, não existe fluxo de massa no topo. Foram obtidos 18 perfis de concentração na coluna num período de 24 dias. Na Figura 1 são mostrados sete perfis de concentração obtidos para a emissão de uma quantidade de $1 \mathrm{~mL}$ do composto tolueno, onde cada curva representa um dia de coleta. O gráfico representa a distância do ponto de emissão (topo da coluna) no eixo da abscissa e a concentração em ppm de massa no eixo das ordenadas. As sete curvas mostradas são os resultados no período de tempo de 1, 3, 6, 10, 13, 14 e 20 dias após a emissão. Para a visualização são mostrados apenas 7 dos 18 perfis amostrados. Nas Figuras 3 a 8 são mostradas três curvas de concentração em cada gráfico. Essas curvas mostram a evolução do valor de concentração na coluna em função do dia de coleta. Os gráficos do experimento demonstram a variação do perfil com o aumento de concentração por difusão na coluna nos primeiros dias (Figuras 3 e 4). Demonstram, ainda, a permanência do composto na forma líquida, na fonte, de 6 a 8 dias após a sua emissão. Também fica claro nos perfis, a inexistência do tolueno na forma líquida após o oitavo dia, quando o valor de concentração começa a decrescer nos pontos próximos do topo (Figuras 5 a 7). Os valores das concentrações, após este dia, também decaem continuamente em toda a coluna, devido ao mecanismo de difusão e à perda de massa para a atmosfera no fundo. Nos perfis, percebe-se, também, a ausência de fluxo do composto no topo da coluna, pois esta foi isolada com o tampão. Portanto, nos primeiros oito dias, ocorre elevação da concentração na coluna pela difusão do composto a partir do ponto de emissão. Após este dia, vê-se a perda de concentração na coluna pela inexistência do composto na forma líquida e da transferência de massa no fundo para a atmosfera, conforme as Figuras 2 a 7. A Figura 8 mostra as curvas para os três pontos mais próximos do topo (10, 30 e $50 \mathrm{~cm}$ ), na qual se pode visualizar melhor o período em que a concentração se mantém constante nessa região (entre o sexto e o oitavo dia), quando o tolueno emitido ainda não evaporou completamente, e o período que os valores de concentrações começam a diminuir nas proximidades do ponto de emissão, quando o composto não deve existir na forma líquida. As curvas dos dias oito e nove mostram essa queda. A Figura 9 mostra a concentração variando com o período de tempo na unidade de dia para o ponto de coleta mais próximo da emissão - o ponto a $10 \mathrm{~cm}$ do topo. A concentração se mantém elevada na região até o oitavo dia. Esse período é suposto a presença do tolueno na forma líquida. A concentração começa a cair na região 


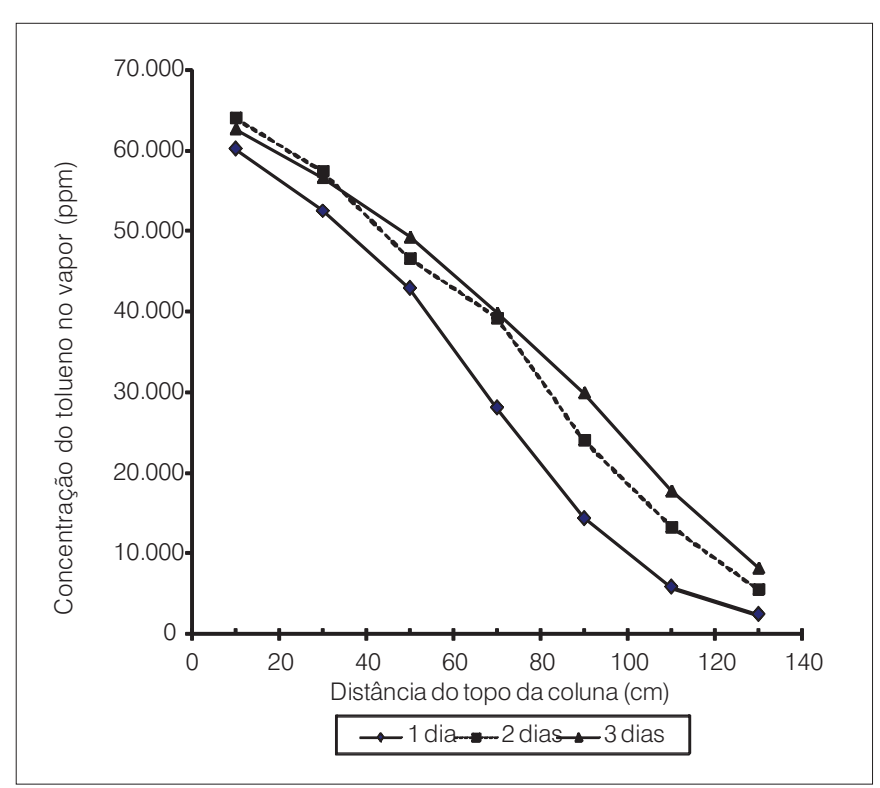

Figura 3 - Perfis de concentração variando com a distância.

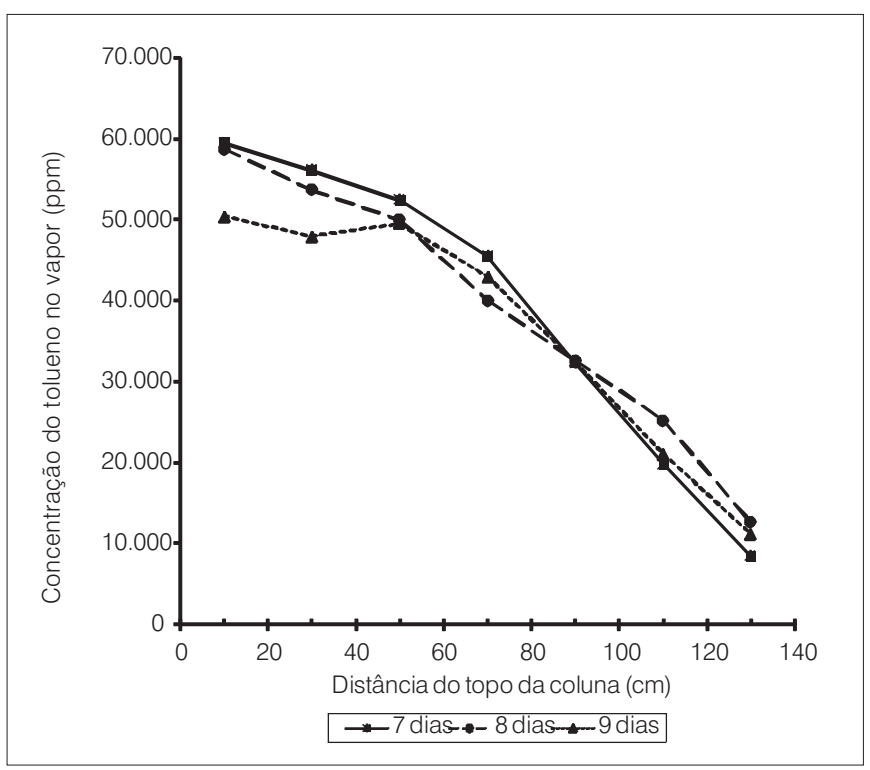

Figura 5 - Perfis de concentração variando com a distância.

após o oitavo dia - o tolueno deve ter evaporado completamente. A Figura 10 mostra a concentração variando com o tempo no período de 15 dias, para os pontos de coleta a 10, 30, 50 e $70 \mathrm{~cm}$.

Uma análise dos resultados experimentais obtidos é realizada com a aplicação dos modelos matemáticos de pluma com dispersão em meio semi-infinito (denominado pluma de solo), fonte de emissão com concentração constante no topo da coluna e condição de fluxo para a atmosfera no comprimento L (denominado modelo de concentração fixa) e um modelo de emissão de uma série de liberações instantâneas com intervalo de um dia entre elas (denominado modelo de puffs). Estes modelos foram mostrados nas Equações 7 , 8 e 9, respectivamente. As Figuras 11, 12, 13 e 14 apresentam os

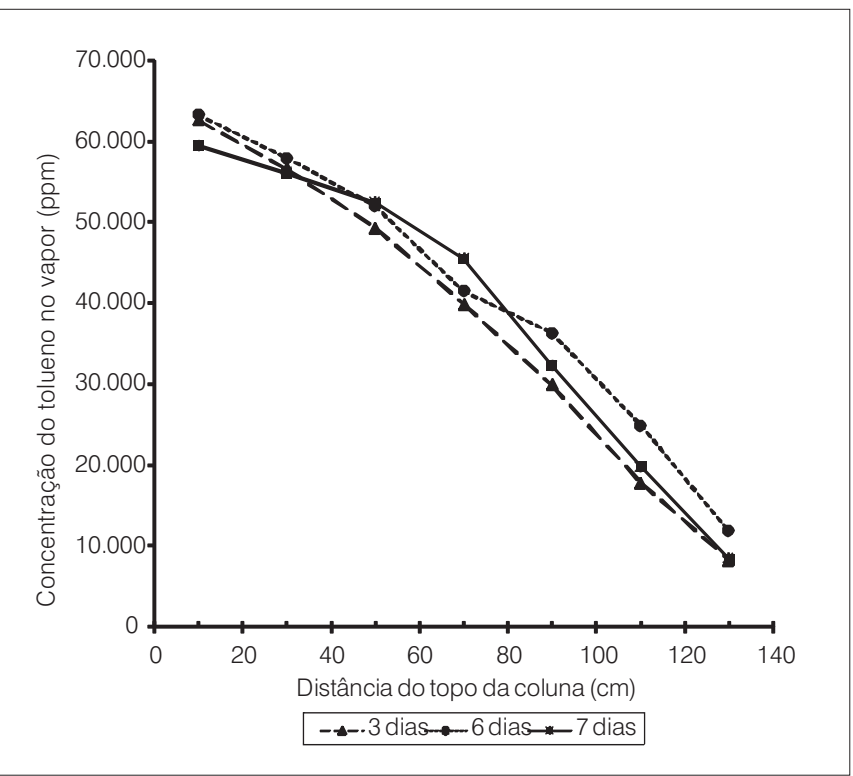

Figura 4 - Perfis de concentração variando com a distância.

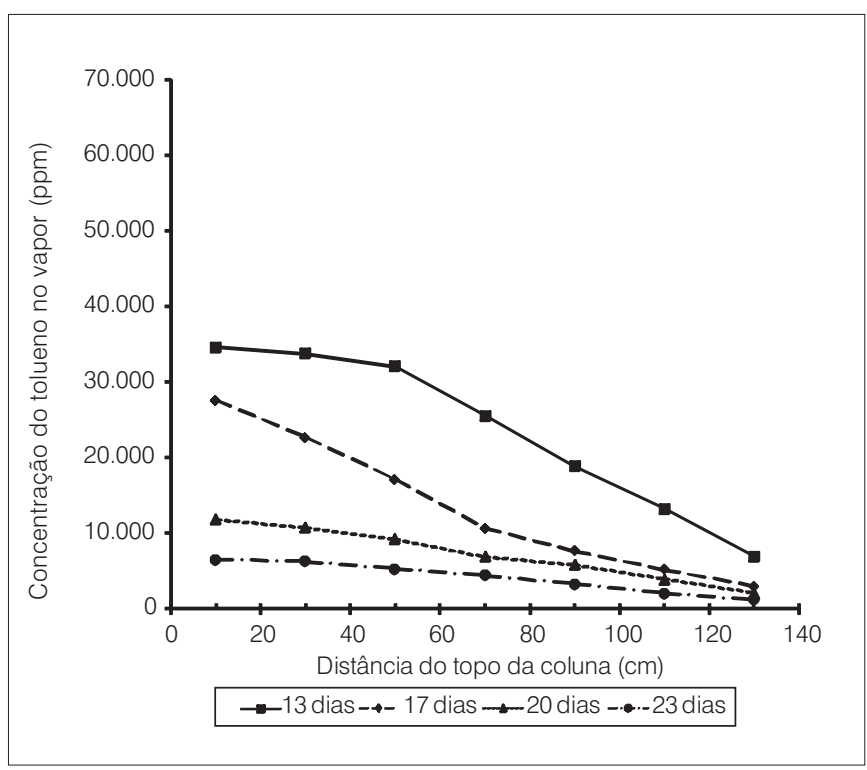

Figura 6 - Perfis de concentração variando com a distância.

perfis de concentração para os dias um, dois, três e seis, respectivamente. Os resultados experimentais são os pontos no gráfico e as simulações numéricas são as linhas curvas com as respectivas legendas. A Tabela 1 apresenta os parâmetros usados nas simulações efetuadas. Estes resultados de simulação parecem demonstrar que a fonte de liberação não permanece contínua, mas está sendo limitada ou esgotada na coluna por algum processo. Esses processos podem ser uma baixa taxa de evaporação do tolueno líquido na fonte devido aos processos de transmissão de calor, uma perda por vazamento na coluna e a retirada de massa no comprimento da coluna pelo mecanismo de transferência global de massa para a atmosfera (condição de fluxo de massa no final da coluna), que serão posteriormente 


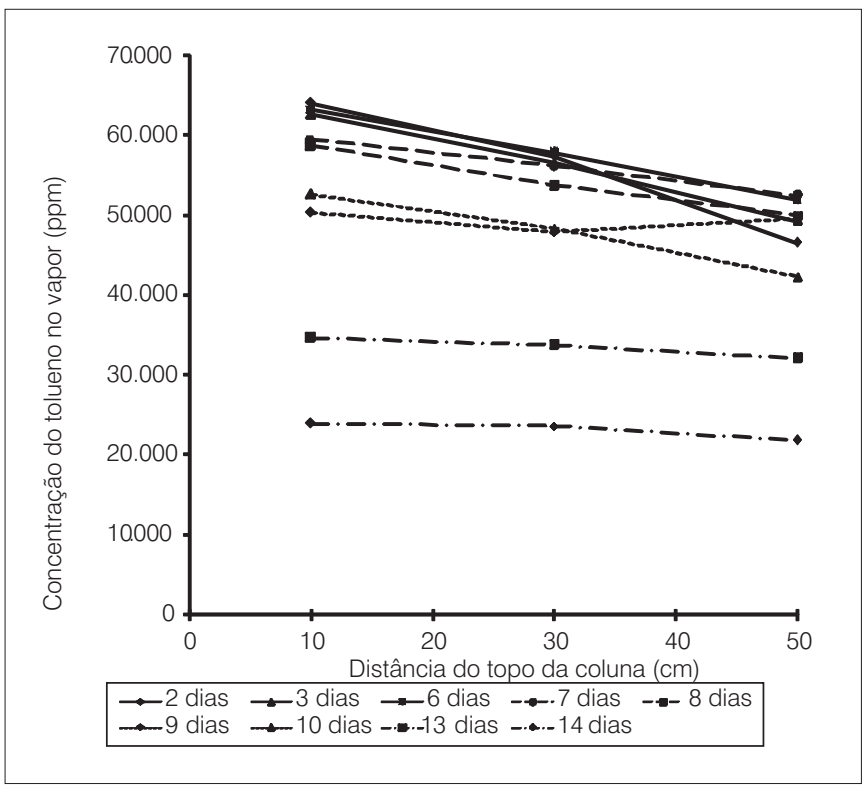

Figura 7 - Perfis de concentração com a distância.

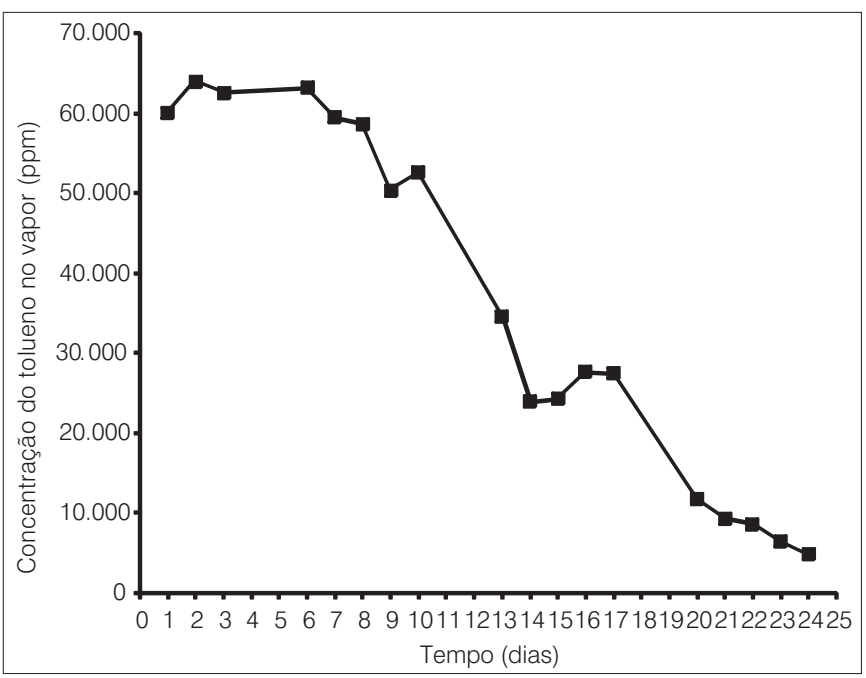

Figura 9 - Variação da concentração pelo tempo, no ponto mais próximo da emissão.

Tabela 1 - Parâmetros usados na simulação numérica.

\begin{tabular}{lc|}
\hline Parâmetro da simulação & Valor do parâmetro \\
\hline Temperatura $\left({ }^{\circ} \mathrm{C}\right)$ & 25,0 \\
\hline Pressão de vapor ( $\mathrm{atm})$ & 0,0244 \\
\hline Coeficiente de difusão no $\mathrm{ar}\left(\mathrm{m}^{2} \mathrm{~s}^{-1}\right)$ & 0,752 \\
\hline Porosidade de solo & 0,42 \\
\hline Porosidade da fase vapor & 0,42 \\
\hline Extensão do puff na fonte (m) & 0,20 \\
\hline $\begin{array}{l}\text { Comprimento do filme estagnado fora da coluna } \\
\text { para avaliação do coeficiente de transferência de } \\
\text { massa (m) }\end{array}$ & 0,15 \\
\hline $\begin{array}{l}\text { Comprimento da coluna (m) } \\
\text { Intervalo de tempo entre os puffs (dia) }\end{array}$ & 1,5 \\
\hline
\end{tabular}

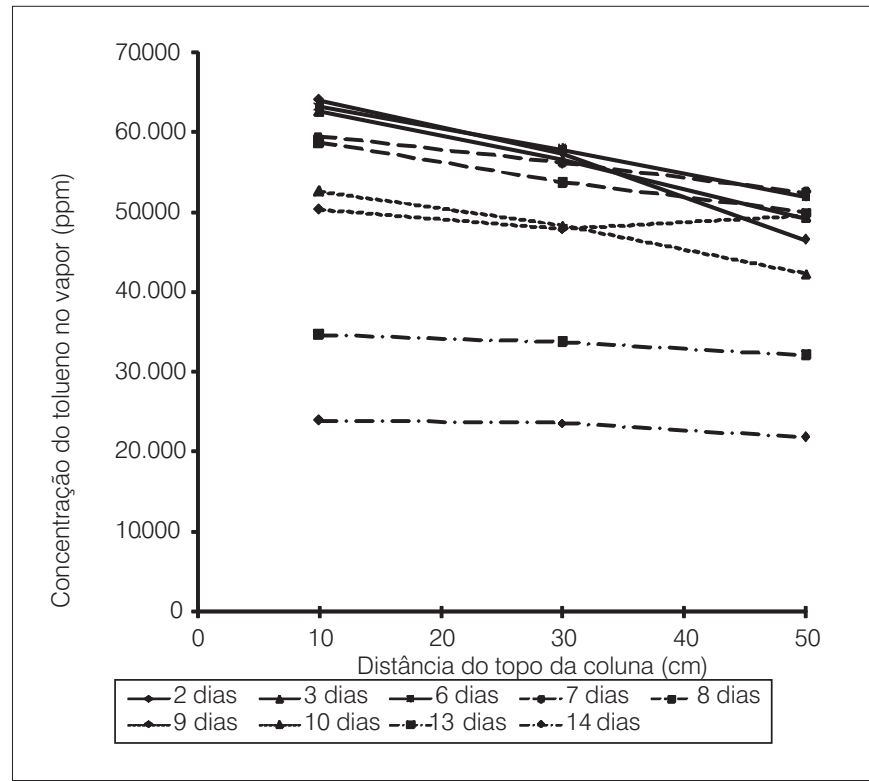

Figura 8 - Variação da concentração do composto nas proximidades do ponto de emissão.

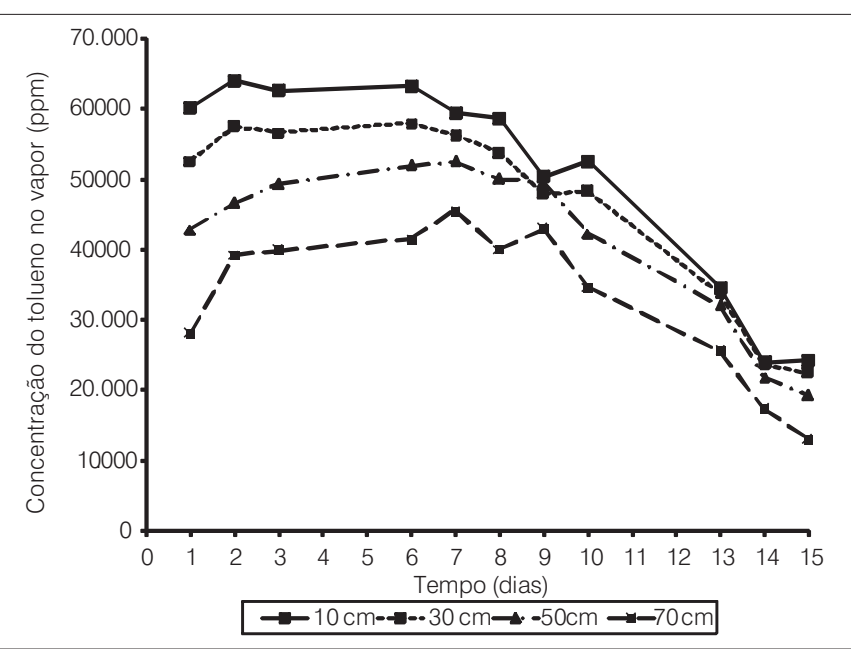

Figura 10 - Variação da concentração pelo tempo, nos quatro pontos mais próximos da emissão.

Tabela 2 - Parâmetros estatísticos obtidos com o modelo de combinação de puffs.

\begin{tabular}{lc|}
\hline Parâmetro estatístico & Valor do parâmetro \\
\hline Desvio fracional & $-0,0772$ \\
\hline Média adimensional & 1,08 \\
\hline $\begin{array}{l}\text { Desvio padrão quadrático nédio adimensional } \\
\text { Número de erros simples positivos obtidos com } \\
\text { o modelo }\end{array}$ & 0,0793 \\
\hline $\begin{array}{l}\text { Média de erros positivos relativos ao valor } \\
\text { medido }\end{array}$ & 12 \\
$\begin{array}{l}\text { Número de erros simples negativos obtidos com } \\
\text { o modelo }\end{array}$ & 0,210 \\
\hline $\begin{array}{l}\text { Média de erros negativos relativos ao valor } \\
\text { medido }\end{array}$ & $-0,0932$ \\
\hline
\end{tabular}




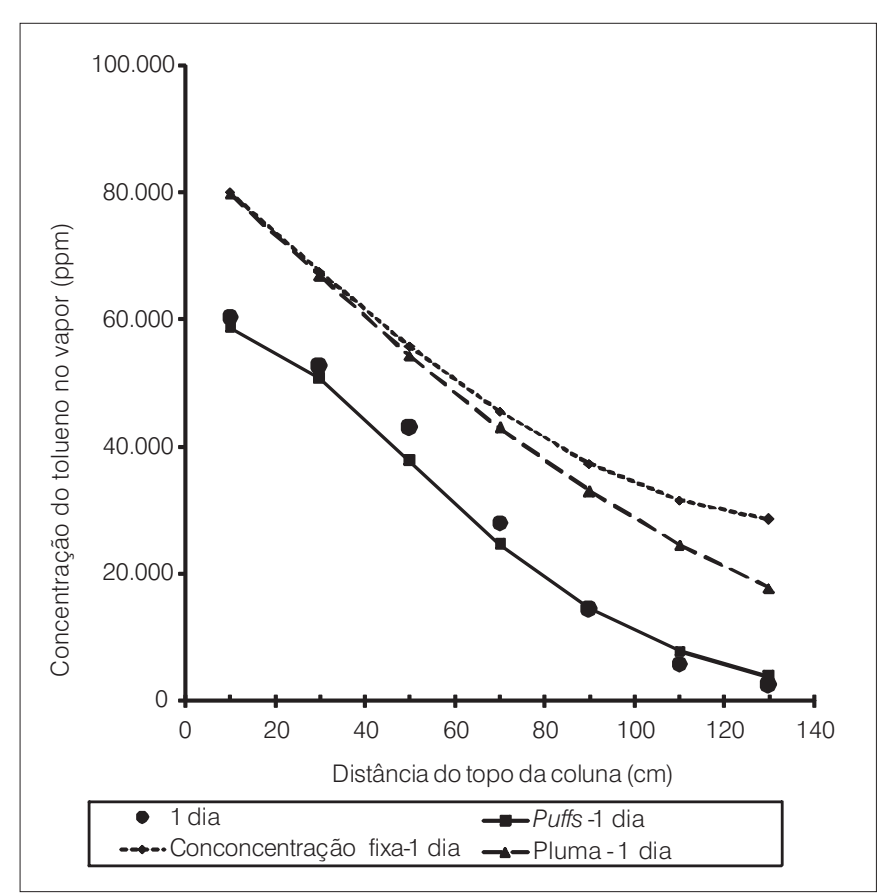

Figura 11 - Modelagem matemática para um dia.

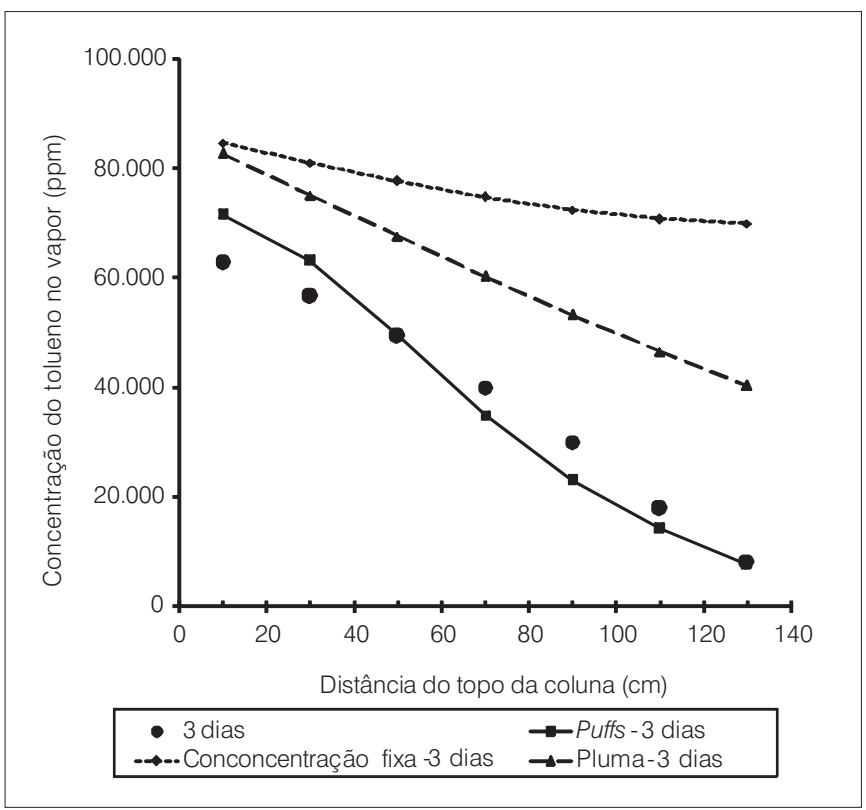

Figura 13 - Modelagem matemática para três dias.

mais investigados. Na Tabela 2 é mostrada uma análise estatística dos valores obtidos para o modelo de combinação de puffs usando índices de avaliação, conforme Hanna; Strimaitis; Chang (1993).

\section{Discussão}

O resultado do teste mostra valores de concentração tendo um perfil estacionário (com a presença da fonte de emissão de tolueno líquido) na coluna até o oitavo dia, quando esses valores começam a baixar

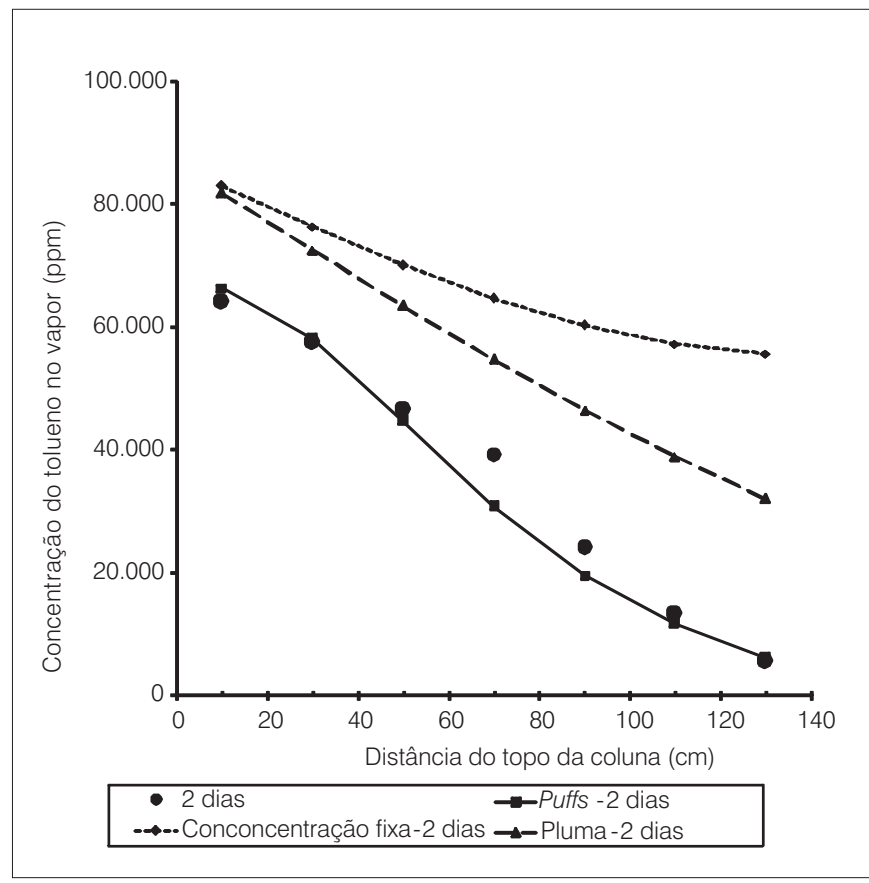

Figura 12 - Modelagem matemática para dois dias.

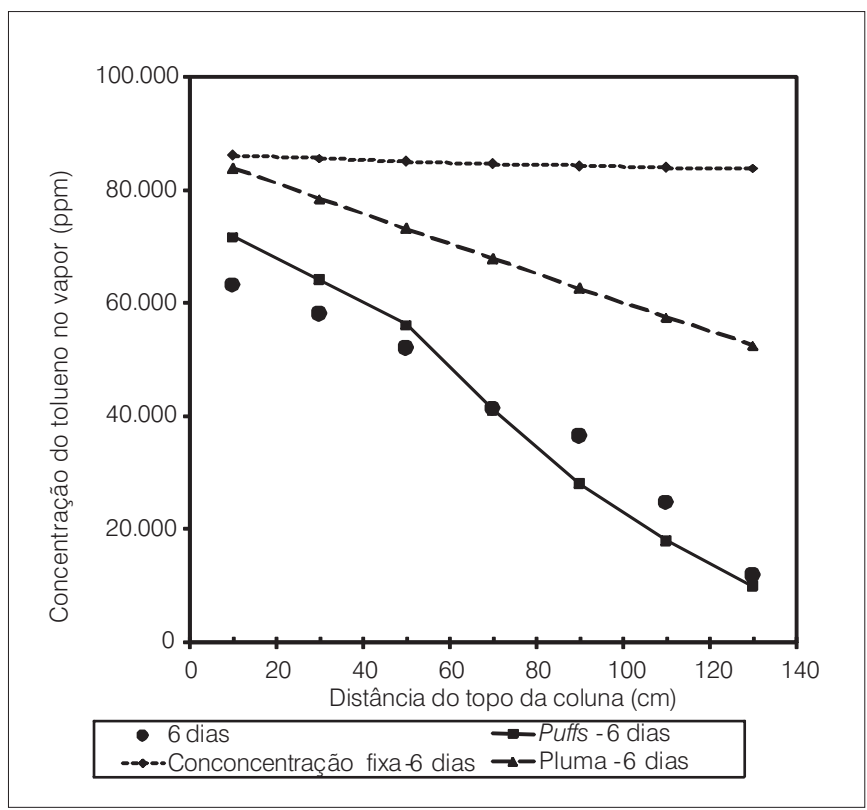

Figura 14 - Modelagem matemática para seis dias.

ao longo da coluna. Isto é, o perfil começa a baixar pela perda da fonte. Os resultados de simulação mostram que a fonte não mantém um valor constante de concentração no ponto de emissão ou ocorre uma perda de tolueno na coluna. O modelo de combinação de liberações instantâneas aplicado mostrou uma boa concordância entre os valores simulados e os experimentais. Uma modelagem deverá ser aplicada para tentar confirmar o comportamento de evaporação do composto no topo da coluna, com a aplicação dos balanços de massa e energia. Mais testes experimentais também estão sendo realizados. 


\section{Conclusões}

Esta pesquisa desenvolveu a avaliação experimental de concentração para compostos de frações de petróleo na zona não saturada do solo, através de uma técnica para o composto tolueno. O procedimento experimental apresentado mostrou-se adequado para obtenção de valores de concentração de compostos hidrocarbonetos na fase gasosa em colunas simulando a zona não saturada do solo. Outros testes deverão ser realizados e modelos matemáticos serão aplicados para obtenção de mais conclusões da pesquisa.

\section{Referências}

ABRIOLA, L.M.; PINDER, G.F. (1985) A Multiphase Approach to the Modeling of Porous Media Contamination by Organic Compounds, 1, Equation Development. Water Resources Research, v. 21, n. 1, p. 11-8.

BEHRENDT, H. (1996) SNAPS/SOIL - water, heat, material transport in soil. Disponível em: <http://ecobas.org/www-server/rem/mdb/snapssoil.html>. Acesso em: 05 dez. 2005.

CORDAZZO, J. (2000) Modelagem e simulação numérica do derramamento de gasolina acrescida de álcool em águas subterrâneas. Dissertação (Mestrado). Florianópolis: Universidade Federal de Santa Catarina.

DOMENICO, P.A.; SCHWARTZ, F.W. (1997) Physical and Chemical Hydrogeology. $2^{\text {nd }}$ ed. New York: John Wiley \& Sons.

FÁVERO, J.L.; MUNIZ, A.R.; SANTOS, R.P.B (2007) Análise teóricoexperimental da dispersão de poluentes líquidos em solos. Engenharia Sanitária e Ambiental, v. 12, n. 4, p. 410.

FETTER, C.W.J. Contaminant hydrogeology. $2^{\text {nd }}$ ed. New York: Macmillan Publishing Co, 1998

GRATHWOHL, P.; WANG, G.; BREDERODE, S; RECKHORN, F. (2003) Volatile organic compounds volatilization from multicomponent organic liquids and diffusion in unsatured porous media, Vadose Zone Journal, v. 2, p. 602-701.

HANNA, S.R.; STRIMAITIS, D. G.; CHANG, J.C. (1983) Hazardous gas model evaluation with field observations. Atmospheric Environment, v. 27, p. 2265-85.

JURY, W.A.; GARDNER, W.R.; GARDNER, W.H. (2005) Soil physics. $6^{\text {th }}$ ed. New York: John Wiley Professional.

MILLINGTON, R.J.; QUIRK, J.M. (1960) Permeability of porous solids. In: $7^{\text {th }}$ International Congress of Soil Science. SSSA, Madison. p. 97-106.

NUNES, C.N.; CORSEUIL, H.X. (2007) Importância do etanol na atenuação natural de águas subterrâneas impactadas por gasolina. Engenharia Sanitária e Ambiental, v. 12, n. 3.

OZISIK, M.N. (1993) Heat conduction. $2^{\text {nd }}$ ed. New York: John Wiley Professional.

PENNER, G.C. (2000) Estudos laboratoriais da contaminação do solo com gasolina com o uso de um detetor de fotoionização. Dissertação (Mestrado). São Carlos: Universidade de São Paulo.

SANTOS, A.H.P. (1998) Simulação composicional para transporte de hidrocarbonetos em aqüiferos. Dissertação (Mestrado). Campinas: Universidade Estadual de Campinas. 